\title{
Resonant tunneling through a macroscopic charge state in a superconducting SET transistor
}

\author{
D.V. Averin \\ Department of Physics, SUNY at Stony Brook, Stony Brook, NY 11794, USA \\ A.N. Korotkov \\ Institute of Nuclear Physics, Moscow State University, 119899 GSP Moscow, Russia \\ A.J. Manninen and J.P. Pekola \\ Department of Physics, University of Jyväskylä, P.O. Box 35, 40351 Jyväskylä, Finland
}

\begin{abstract}
We predict theoretically and observe in experiment that the differential conductance of a superconducting SET transistor exhibits a peak which is a complete analogue in a macroscopic system of a standard resonant tunneling peak associated with tunneling through a single quantum state. In particular, in a symmetric transistor, the peak height is universal and equal to $e^{2} / 2 \pi \hbar$. Away from the resonance we clearly observe the co-tunneling current which in contrast to the normal-metal transistor varies linearly with the bias voltage.
\end{abstract}

PACS numbers: 73.40.Gk, 74.50.+r

Typeset using REVTEX 
Charging effects in systems of small Josephson junctions are quite well understood by now - see, e.g., [1.2]. Interest, however, has been focused mostly on the interplay between the charging effects and Cooper pair transport, which can be described in generic terms as the quantum dynamics of the Josephson phase difference. The aim of this work is to study the quasiparticle transport in a superconducting SET transistor - a system of two junctions connected in series (see inset in Fig. 1). We show that the BCS singularity in the density of states of superconducting electrodes of the junctions brings about several interesting new features of quasiparticle transport. Most notably, in the vicinity of the threshold voltage $V_{t}$ for classical tunneling the quasiparticle transport is identical to resonant tunneling through a single macroscopic quantum state of the transistor.

In this work, we study the low voltage regime, $V<V_{t}$, where the quasiparticles do not have enough energy to enter the central electrode of the transistor and can traverse it only by quantum tunneling through the energy barrier created by the charging energy of the central electrode. The effects of the superconducting density of states in the classical sequential tunneling were discussed recently in Ref. [3]. The dominant contribution to the current $I$ in the regime of quantum tunneling comes from the so-called inelastic co-tunneling, the process in which two different electrons tunnel simultaneously in the two junctions of the transistor, and can be written [4] as:

$$
\begin{gathered}
I=e(\Gamma(V)-\Gamma(-V)) \\
\Gamma(V)=\frac{8 \pi}{\hbar} \int \prod_{j=1}^{4}\left[d \epsilon_{j} N_{j}\left(\epsilon_{j}\right)\left(1-f\left(\epsilon_{j}\right)\right)\right] \delta\left(e V-\sum_{j=1}^{4} \epsilon_{j}\right) \\
\left|T_{1} T_{2}\right|^{2}\left|\frac{1}{E_{1}+\epsilon_{1}+\epsilon_{2}-2 \Delta}+\frac{1}{E_{2}+\epsilon_{3}+\epsilon_{4}-2 \Delta}\right|^{2} .
\end{gathered}
$$

Here $\epsilon_{1}, \epsilon_{2}$ and $\epsilon_{3}, \epsilon_{4}$ are the energies of the states between which electrons are transferred in the first and second junction, respectively, $T_{1,2}$ and $N_{j}\left(\epsilon_{j}\right)$ are the corresponding tunneling amplitudes and densities of states in the electrodes, $f(\epsilon)$ is the Fermi distribution function, $V$ is the bias voltage, and we have assumed for simplicity that all electrodes have the same energy gap $\Delta$. 
We restrict our attention to the case of low temperatures, $T \ll \Delta$, when the nonvanishing quasiparticle current exists only at large voltages, $V>4 \Delta / e$, sufficient for the creation of quasiparticles in the two junctions. In this voltage range the energies $E_{1,2}$ of the intermediate charge states in eq. (11) are:

$$
E_{1}=E_{C}-\lambda(e V-4 \Delta)-\frac{e Q_{0}}{C_{\Sigma}}, \quad E_{2}=E_{C}-(1-\lambda)(e V-4 \Delta)+\frac{e Q_{0}}{C_{\Sigma}},
$$

where $E_{C}=e^{2} / 2 C_{\Sigma}$ with $C_{\Sigma}=C_{1}+C_{2}+C_{g}$ denoting the total capacitance of the central electrode of the transistor, $\lambda=\left(C_{2}+C_{g}\right) / C_{\Sigma}$ gives the fraction of the bias voltage that drops across the first junction, and $Q_{0}=e \times\left\{V_{g} C_{g} / e+\Delta(2 \lambda-1) / E_{C}\right\}$ with $\{x\} \equiv x-[x+1 / 2]$ can be interpreted as the charge induced by the gate voltage $V_{g}$ into the central electrode.

Integrating over $\epsilon_{1}, \epsilon_{2}$ at fixed $\epsilon_{1}+\epsilon_{2}$ and similarly over $\epsilon_{3}, \epsilon_{4}$ we can express the cotunneling rate (1) in terms of the "seed" $I-V$ characteristics $I_{j}(U), j=1,2$, of the two junctions at a fixed voltage $U$ across a single junction and no charging effects:

$$
\begin{gathered}
\Gamma(V)=\frac{\hbar}{2 \pi e^{2}} \int d \epsilon \frac{I_{1}(\epsilon / e)}{1-\exp (-\epsilon / T)} \frac{I_{2}(V-\epsilon / e)}{1-\exp (-(e V-\epsilon) / T)}|M|^{2}, \\
M=\frac{1}{E_{1}+\epsilon-2 \Delta}+\frac{1}{E_{2}+e V-\epsilon-2 \Delta} .
\end{gathered}
$$

¿From eq. (3) we see directly that a jump of the quasiparticle current $I_{j}(U)$ at $U=2 \Delta / e$ in superconducting junctions changes the voltage dependence of the co-tunneling current for $V$ close to $4 \Delta / e$ from cubic $\left(\Gamma(V) \propto V^{3}\right.$ for a normal-metal transistor [4]) to linear. Indeed, for $T \ll \Delta$ we can approximate $I(U)$ near the threshold $U=2 \Delta / e$ as (see, e.g., [5]):

$$
I(U)=I_{j} \Theta(U-2 \Delta / e), \quad I_{j}=\frac{\pi \Delta}{2 e R_{j}},
$$

where $R_{j}$ is the normal-state tunnel resistance of the $j$ th junction. Equations (3) and (4) give for low temperatures and $e V-4 \Delta \ll \Delta, E_{C}$ :

$$
I(V)=e \Gamma(V)=\frac{\hbar I_{1} I_{2}}{2 \pi}\left(\frac{1}{E_{1}}+\frac{1}{E_{2}}\right)^{2}\left(V-\frac{4 \Delta}{e}\right) .
$$

When the bias voltage approaches the threshold $V_{t}$ of classical sequential tunneling, where one of the energy barriers $E_{j}$ vanishes, the co-tunneling current grows and crosses over into 
the current carried by sequential tunneling, in which quasiparticles traverse the transistor by two independent jumps across the two junctions. It is known that the energy width of the crossover region between the co-tunneling and sequential tunneling is determined by the lifetime broadening of the intermediate charge states $E_{1,2}$ [6]9. If the gate voltage is not close to the special point $Q_{0}=(1 / 2-\lambda) e$ where $E_{1,2}$ vanish simultaneously (the situation that corresponds to the maximum threshold voltage $\left.V_{t}=\left(4 \Delta+2 E_{C}\right) / e\right)$, then the current through one intermediate state, for instance $E_{1} \equiv E$, dominates near the tunneling threshold. The current in the transition region can be described in this situation by simply adding the lifetime broadening $\gamma$ of the intermediate state in eq. (3) for the co-tunneling rate 10,11]:

$$
M=\frac{1}{E+\epsilon-2 \Delta+i \gamma}, \quad \gamma=\frac{\hbar}{2 e}\left[I_{1}\left(\frac{\epsilon}{e}\right) \operatorname{coth}\left(\frac{\epsilon}{2 T}\right)+I_{2}\left(\frac{e V-\epsilon}{e}\right) \operatorname{coth}\left(\frac{e V-\epsilon}{2 T}\right)\right] .
$$

(This simple approach neglects only the renormalization of $E$ and $\gamma$ significant at temperatures exponentially small on the scale of $E_{C}$ [11].)

Combining eqs. (2), (3), (田), and (6) we can calculate the differential conductance of the transistor at low temperatures:

$$
G=\frac{d I}{d V}=\frac{\hbar I_{1} I_{2}}{2 \pi}\left[\frac{\lambda}{\left(E_{0}-\lambda(e V-4 \Delta)\right)^{2}+\delta^{2}}+\frac{1-\lambda}{\left(E_{0}+(1-\lambda)(e V-4 \Delta)\right)^{2}+\delta^{2}}\right],
$$

where $E_{0}=\left(e / 2-\left|Q_{0}\right|\right) e / C_{\Sigma}$ is the Coulomb energy barrier at $V=4 \Delta / e$, and $\delta=\hbar\left(I_{1}+\right.$ $\left.I_{2}\right) / 2 e$ is the energy width of the charge state due to tunneling. If we use the second equation in the expression (4) we see that $\delta=\pi \hbar\left(R_{1}^{-1}+R_{2}^{-1}\right) \Delta / 4 e^{2}$. Since the ideology of co-tunneling is applicable only to junctions with small tunnel conductance, $R^{-1} \ll e^{2} / h$, this means that the width of the charge state is small, $\delta \ll \Delta$, and eq. (7) describes the narrow conductance peak located at the threshold $V_{t}$ of classical tunneling $\left(e V_{t}=4 \Delta+E_{0} / \lambda\right)$. This peak corresponds to the rapid current rise from almost zero to $I_{1} I_{2} /\left(I_{1}+I_{2}\right)$ at $V=V_{t}$. The maximum conductance is achieved when $E_{0}=0$ (i.e., when the tunneling threshold reaches minimum) and $V=V_{t}=4 \Delta / e$ :

$$
G=\frac{d I}{d V}=\frac{e^{2}}{2 \pi \hbar} \frac{4 I_{1} I_{2}}{\left(I_{1}+I_{2}\right)^{2}} .
$$


Equation (8) shows that in a symmetric transistor, where $I_{1}=I_{2}$, the differential conductance reaches the absolute maximum $e^{2} / 2 \pi \hbar$ which is independent of $\Delta, E_{C}$, or the junction resistance $R$. This universality is similar to that of the resonant tunneling through a single microscopic quantum state, and is quite remarkable in view of the fact that in the present context the quantum state is the macroscopic charge state of the central electrode of the transistor.

If the energy barrier $E_{0}$ is large on the scale of the width $\delta$ of the charge state, $\delta$ starts to increase with increasing $E_{0}$, i.e. increasing threshold voltage $V_{t}$. The conductance peak can be described analytically in this regime by retaining only the first, resonant, term in eq. ([7), and taking into account that the peak width $\delta$ depends then on its position $V_{t}$ through the dependence on $V_{t}$ of the contribution of the current $I_{2}$ through the second junction to $\delta: I_{2}=I_{2}\left(V_{t}-2 \Delta / e\right)$.

The shape of the conductance peak in a symmetric transistor (with $R_{1}=R_{2}$, and $\lambda=1 / 2$ ) calculated numerically from the eqs. (2), (3), and (6) without the approximation (四) or restrictions on $E_{0}$ is shown in Fig. 1. We see that this, more accurate, calculation preserves all the qualitative features of the simple analytical expression (7): maximum conductance is $e^{2} / 2 \pi \hbar$ when $E_{0}=0$ and decreases to approximately half this value at nonzero $E_{0}$.

For the results discussed above to be valid, the lifetime broadening of the resonant charge state should not only be much smaller than the superconducting gap $\Delta$, but also much smaller than the typical energy distance (on the order of $E_{C}$ ) to the excited charge states of the central electrode of the transistor. The condition for this is:

$$
\alpha \equiv \frac{\Delta}{E_{C}} \frac{\pi \hbar}{e^{2}}\left(R_{1}^{-1}+R_{2}^{-1}\right) \ll 1
$$

It is important that this condition can be violated not only when the normal-state junction conductances are large, but also when the energy gap $\Delta$ is large in comparison to the charging energy $E_{C}$. If it is indeed violated, the charging effects are eventually washed out by quantum fluctuations and the current rise at $V=4 \Delta / e$ becomes infinitely sharp 
(provided that the singularity of the density of states at the energy gap $\Delta$ is not smeared out by some internal mechanism like spin-flip scattering).

To test these predictions experimentally we fabricated and measured four superconducting SET transistors with differing parameters. The transistors were fabricated by electron beam lithography on oxidised silicon by the standard shadow evaporation technique using aluminum electrodes and aluminum oxide junction barriers. The geometry of each of the transistors was such that the length of the central island was $1 \mu \mathrm{m}$, its width was $80-120$ $\mathrm{nm}$, and the overlap at the two ends of the island with the external electrodes was nominally $70 \mathrm{~nm}$. The gate electrode was simply a $100 \mathrm{~nm}$ wide finger, pointing orthogonally to the center of the island at a distance of about $0.5 \mu \mathrm{m}$. The gate capacitance was about $0.02 \mathrm{fF}$.

Tunnel resistance $R$ of the transistor junctions was measured from the large-voltage asymptote of the $I-V$ characteristic of the transistor assuming equal resistances of the two junctions. Although we did not carry out any systematic study of how symmetric the transistors were, we checked from the gate voltage dependence of the threshold voltage $V_{t}$ that sample 1 had equal parameters to within $30 \%$, and we do not expect the other transistors to be worse in this respect since their dimensions were larger than in sample 1 . The charging energy $E_{C}$ was measured as a half of the amplitude of the $V_{t}$ modulation by the gate voltage, and $\Delta$ can be obtained from the onset of the current at $4 \Delta / e$. All these parameters of the four samples are shown in Table 1, together with the combined parameter $\alpha$ defined in eq. (9) as a small parameter of the present theory.

Measured $I-V$ characteristics and traces of the differential conductance of sample 1 as a function of the bias voltage $V$ are shown in Fig. 2 for several values of the gate voltage. The curves agree qualitatively with the predictions of the theory described above. The differential conductance has a narrow peak of the roughly correct width at the threshold of classical tunneling. The height of the peak away from the resonance is slightly below one-half of $e^{2} / 2 \pi \hbar$. The main discrepancy between the experimental results (Fig. 2b) and the simple model calculations (Fig. 1) is that at resonance the conductance does not reach the ideal maximum value $e^{2} / 2 \pi \hbar$ but rather is about one half of this value. Although 
the asymmetry of junction resistances contributes according to eq. (8) to suppression of the resonance conductance, the actual asymmetry of our transistors was too small to account for the observed magnitude of this suppression. This discrepancy can be qualitatively explained by the fact that in close similarity to regular resonant tunneling, the resonant tunneling through the macroscopic charge state discussed in this work is very sensitive to all sources of inelastic scattering. For instance, we checked numerically that weak spin-flip scattering with the rate $\tau_{s}^{-1}=0.01 \Delta / \hbar$ is sufficient to suppress the resonant conductance peak to the level found in the experiment. The model with spin-flip scattering, however, did not reproduce correctly the full shape of the observed conductance curves and we think at the moment that in our transistor the resonance is suppressed by a combination of several inelastic scattering mechanisms including fluctuations of the bias and gate voltage (associated with the finite impedance of the voltage leads), and inelastic tunneling through the tunnel barriers. We could not characterise quantitatively all these sources of inelastic scattering, and therefore did not attempt to find a theoretical fit to the curves in Fig. 2.

The results of measurements for all four samples are summarized in the right-hand-part of Table 1, which shows two characteristic values of the differential conductance in units of $e^{2} / 2 \pi \hbar$ : (i) $G_{0, \exp }$, the conductance at bias voltage just above $4 \Delta / e$ and at gate voltage that corresponds to the maximum threshold voltage $V_{t}$, and (ii) $G_{1, \text { exp }}$, the peak conductance at resonance (when $V_{t}$ reaches minimum). Variation of the peak conductance $G_{1}$ with the tunnel resistance $R$ and charging energy $E_{C}$ described by Table 1 confirms that when the relative width of the charge states of the transistor (characterised by the parameter $\alpha$ of eq. (9)) becomes considerable, $G_{1}$ increases gradually beyond $e^{2} / 2 \pi \hbar$. At large $\alpha$, when $G_{1}$ is much larger than $e^{2} / 2 \pi \hbar$, the charging effects are completely washed out by the quantum fluctuations of charge on the central electrode of the transistor and the differential conductance becomes insensitive to the gate voltage. This case is approached by sample 4 with the largest $\alpha$ in which $V_{t}$ is practically independent of the gate voltage, and we could assign only one value of the characteristic conductance to this sample.

When $\alpha$ is small and the charging effects are well-pronounced, the threshold conductance 
$G_{0}$ originates only from the process of co-tunneling, and is much smaller than the peak conductance $G_{1}$. It can be calculated from eq. (5) which predicts that at $V=4 \Delta / e$ the co-tunneling conductance of the superconducting SET transistor should increase abruptly to a finite, voltage-independent level which also does not depend on temperature at low temperatures. This behavior is indeed found in our three samples with larger tunnel resistances. Figure 3 shows for example the data for sample \# 2. At gate voltages which correspond to the thresholds $V_{t}$ close to maximum we see the kink in the $I-V$ curves and the step in the $d I / d V$ at the onset of the quasiparticle current at $V=4 \Delta / e$. (For other values of the gate voltage small current peaks due to Cooper pair tunneling that are visible in Figs. 2a and 3, overlap with the onset of quasiparticle current and do not allow to identify the conductance jump.) The data shown in Figs. 2 and 3 were taken at temperature of about $100 \mathrm{mK}$. We checked that the jump in the quasiparticle conductance is practically temperature-independent for temperatures up to $0.4 \mathrm{~K}$.

Table 1 contains a comparison between the observed co-tunneling conductance $G_{0, \exp }$ and $G_{0, t h e o r y}$ calculated from eq. (5) under the assumption of a symmetric transistor. Taking into account that any asymmetry of the junction tunnel resistances increases $G_{0}$ we can say that the agreement between $G_{0, \exp }$ and $G_{0, \text { theory }}$ is reasonable.

In summary, we proposed theoretically and confirmed in experiment that the quasiparticle transport in a superconducting SET transistor in the vicinity of the tunneling threshold can be described as resonant tunneling through a macroscopic charge state of the central electrode of the transistor. The maximal differential conductance associated with this process is $e^{2} / 2 \pi \hbar$, while the width of the resonance is determined by the lifetime broadening of the charge states of the transistor. For gate voltages away from the resonance we observed very clearly the co-tunneling current which exhibits linear (in contrast to cubic of the normal-metal case) dependence on the bias voltage.

We gratefully acknowledge financial support of the Academy of Finland and US AFOSR, and Mikko Leivo for help with the experiments. 


\section{REFERENCES}

[1] D.V. Averin and K.K. Likharev, in: "Mesoscopic Phenomena in Solids", ed. by B.L. Altshuler et al. (Elsevier, Amsterdam, 1991), p. 173.

[2] M. Tinkham, "Introduction to Superconductivity", (McGraw-Hill, New York, 1996), Ch. 7.

[3] A.N. Korotkov, Appl. Phys. Lett. 69, 2593 (1996).

[4] D.V. Averin and A.A. Odintsov, Phys. Lett. A 140, 251 (1989).

[5] K.K. Likharev, "Dynamics of Josephson Junctions and Circuits" (Gordon and Breach, New York, 1986), Ch. 2.

[6] A.N. Korotkov, D.V. Averin, K.K. Likharev, and S.A. Vasenko, in: "Single-Electron Tunneling and Mesoscopic Devices", ed. by H. Koch and H. Lübig (Springer, Berlin, 1992).

[7] Yu.V. Nazarov, J. Low Temp. Phys. 90, 77 (1993).

[8] P. Lafarge and D. Esteve, Phys. Rev. B 48, 14309 (1993).

[9] C. Pasquier, U. Meirav, F.I.B. Williams, and D.C. Glattli, Phys. Rev. Lett. 70, 69 (1993).

[10] D.V. Averin, Physica B 194-196, 979 (1994).

[11] H. Schoeller and G. Schön, Phys. Rev. B 50, 18436 (1994). 


\section{FIGURES}

Calculated bias-voltage dependence of the differential conductance of a symmetric superconducing SET transistor with junction resistance $R=20 \hbar / e^{2}$. The curves are plotted for several values of the gate voltage, i.e., the charge $Q_{0}$ induced on the central electrode

of the transistor, that correspond to several charging energy barriers $E_{0}$ for tunneling: $E_{0} /\left(\hbar \Delta / R e^{2}\right)=0 ; 1 ; 3 ; 6 ; 10$. The induced charge $Q_{0}$ can not be close to 0 . The inset shows the equivalent circuit of the SET transistor.

Measured (a) $I-V$ characteristics, and (b) bias-voltage dependence of the differential conductance of sample 1 for several gate voltages. The traces shown with thick lines in (b) correspond to the $I-V$ curves presented in (a). For clarity, the features due to the current peaks associated with the Cooper-pair tunneling that are visible in (a) have been omitted in (b). For discussion see text.

Measured $I-V$ characteristics of sample 2 for several gate voltages. The inset shows the differential conductance in the vicinity of the gap edge $V=4 \Delta / e$ for gate voltages which correspond to the two largest tunneling thresholds. The conductance jump at $V=4 \Delta / e$ is due to the co-tunneling. 


\section{TABLES}

\begin{tabular}{|c|c|c|c|c|c|c|c|}
\hline \hline sample & $R(\mathrm{k} \Omega)$ & $E_{C}(\mathrm{meV})$ & $\Delta(\mathrm{meV})$ & $\alpha$ & $G_{0, \exp }$ & $G_{0, \text { theory }}$ & $G_{1, \exp }$ \\
\hline 1 & 206 & 0.35 & 0.22 & 0.08 & $3.1 \cdot 10^{-3}$ & $1.6 \cdot 10^{-3}$ & .5 \\
\hline 2 & 152 & 0.15 & 0.21 & 0.24 & 0.032 & 0.014 & 0.9 \\
\hline 3 & 65 & 0.15 & 0.20 & 0.55 & 0.096 & 0.086 & 1.6 \\
\hline 4 & 52 & 0.08 & 0.23 & 1.44 & - & - & 4.0 \\
\hline \hline
\end{tabular}

TABLE I. Parameters of the four studied SET transistors. Conductances in the last three columns are shown in units of $e^{2} / 2 \pi \hbar$. 


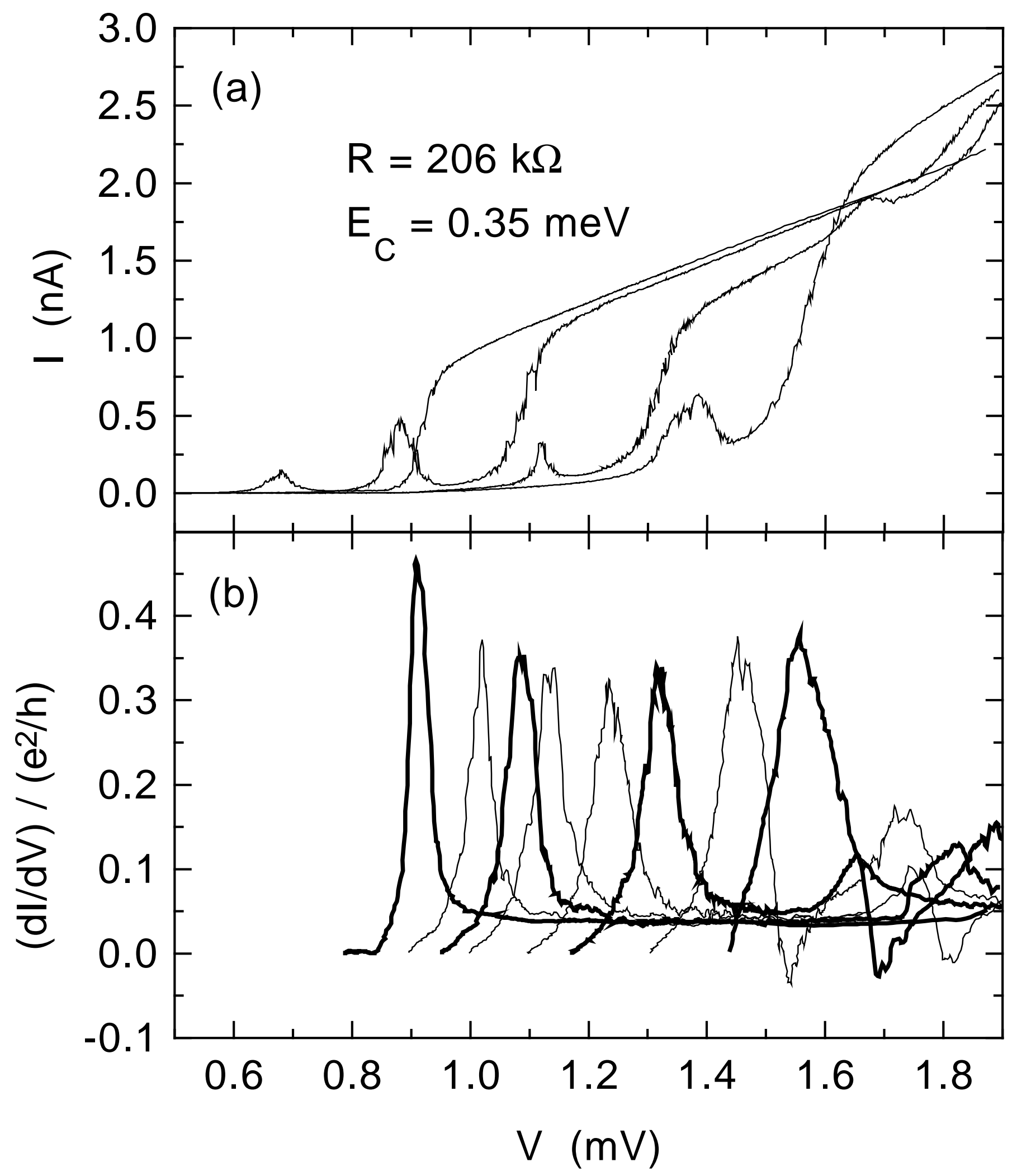




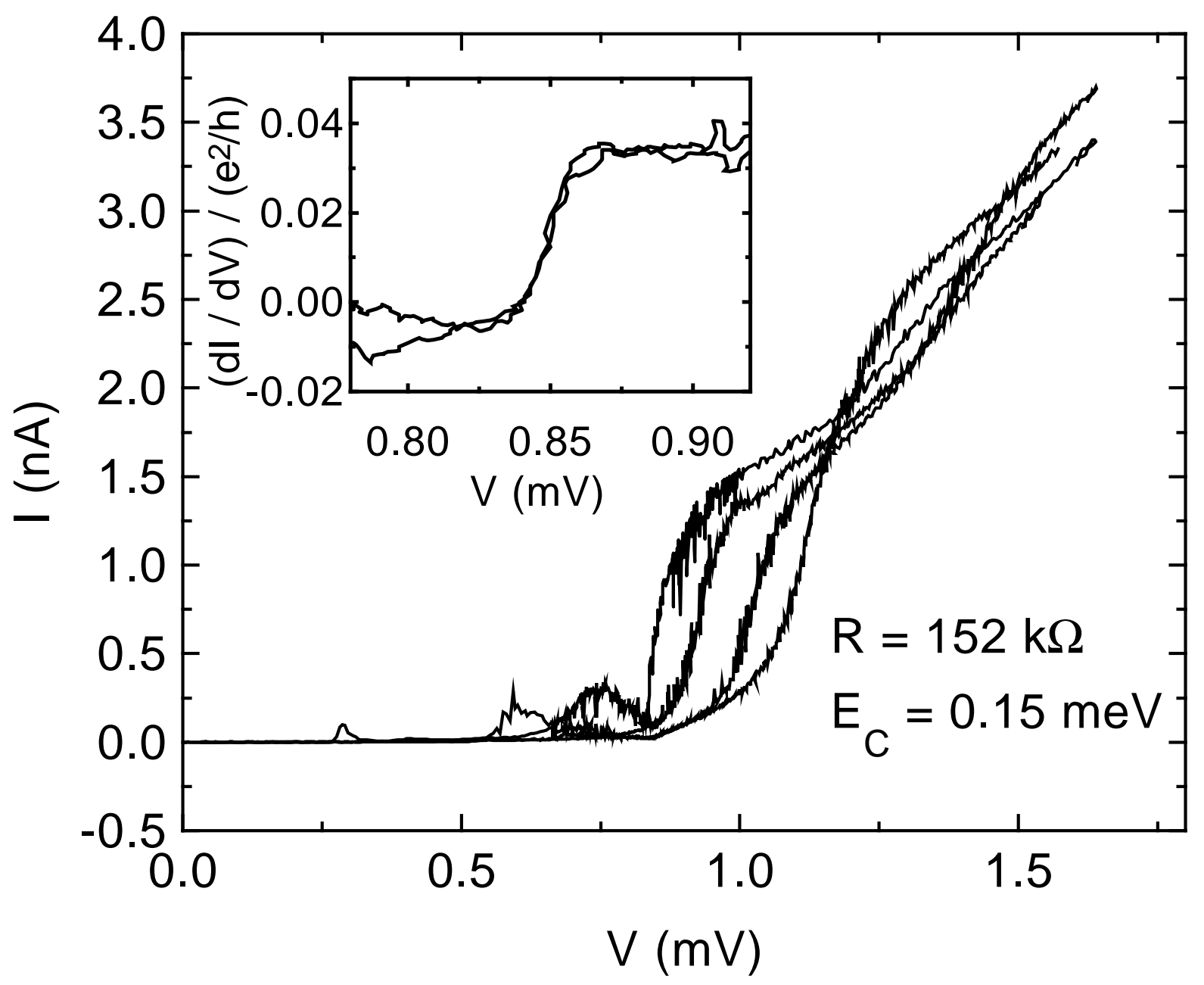

